\title{
Optokinetic stimulation influences the disturbed perception of body orientation in spatial neglect
}

\author{
Hans-Otto Karnath
}

\begin{abstract}
The effect of optokinetic stimulation on the disturbed perception of body orientation in three patients with right brain damage and spatial neglect was examined. The patients were asked to direct a laser point to the position which they felt lay exactly "straight ahead" of their bodies' orientation. Without stimulation they localised the body's sagittal midplane markedly to the right of the objective orientation. The patients' horizontal displacement of the sagittal midplane was reduced by a movement of the surround to the left and worsened by a movement to the right. The findings are consistent with those found in patients with spatial neglect using vestibular and neck proprioceptive stimulation. They show that visual input, together with vestibular and neck proprioceptive input, is used for computing a central representation of egocentric space. In spatial neglect this coordinate transformation works with a systematic error and deviation of the spatial reference frame to the ipsilesional side. The positive effect of optokinetic stimulation in patients with spatial neglect is interpreted with a "correction" of the neural coordinate transformation process by producing asymmetric input at the sensory organs of the contributing channels.
\end{abstract}

(F Neurol Neurosurg Psychiatry 1996;60:217-220)

Keywords: neglect; optokinetic stimulation; egocentric spatial reference frame; brain damage

Heilman et $a l^{1}$ reported that five patients with right hemispheric lesions and left sided neglect showed a deviation to the ipsilateral half of space when they were requested to point to an imaginary spot in space perpendicular to the midline of the chest with their eyes closed. Performing a similar task, patients with neglect did not benefit from seeing the orientation of their bodies. ${ }^{2}$ The pointing errors toward the ipsilesional side of a horizontal slit positioned at the centre of a board were the same as when their bodies were shielded from view. The findings show a disturbed percep- tion of body orientation in patients with neglect-namely, a disparity of subjective and objective body orientation.

This horizontal displacement of sagittal midplane to the right-that is, the disturbed perception of "straight ahead" body orientation, could be compensated for either by neck muscle vibration or by caloric vestibular stimulation on the left side. ${ }^{3}$ Stimulation on the right side led to a transient worsening of the disturbed perception of body orientation. The results suggest that the afferent information from these different input channels contribute to the computation of egocentric spatial coordinates used to determine body position in space. A disturbance of this transformation process seems to lead to the characteristic symptomatology of spatial neglect-that is, the deficient exploration of and response to stimuli located in the contralesional part of space.

In normal subjects Brecher et $\mathrm{al}^{4}$ found that visual input, as a further source, contributes to the central representation of egocentric space. The authors asked their subjects to walk backward and forward in a straight line inside an optokinetic drum rotating slowly in a horizontal direction. The subjects consistently deviated from a straight path toward the direction of the drum motion without being aware of it. When the subjects were asked to set a luminous line to apparent "straight ahead", during rotation of the drum the authors found a deviation of the judgements towards the direction of drum motion.

With the above explanation of spatial neglect, the findings of Brecher et al $\mathrm{l}^{4}$ predict a positive effect of optokinetic stimulation on the patients' disturbed perception of body position in space. The present experiment tests this prediction.

\section{Materials and methods PATIENTS}

Three patients with neglect were examined. The table gives the demographic and clinical data. Brain lesions were documented by CT or MRI. Neuropsychological examination including confrontation testing, copying, line bisection, cancellation, and picture comparison showed a pronounced left sided spatial neglect in each of the patients. Goldmann perimetry showed normal binocular visual fields. 
Demographic and clinical data of the patients with neglect (N1-3) and the brain damaged controls (C1-6)

\begin{tabular}{|c|c|c|c|c|c|}
\hline Patient & Age/sex & Aetiology & $\begin{array}{l}\text { Location of lesion } \\
\text { (side) }\end{array}$ & $\begin{array}{l}\text { Paresis } \\
\text { (side) }\end{array}$ & $\begin{array}{l}\text { Time since } \\
\text { first clinical } \\
\text { symptoms (days) }\end{array}$ \\
\hline N1 & $70 / \mathrm{M}$ & $\begin{array}{l}\text { OP grade } \\
\text { IV glioma }\end{array}$ & $\mathbf{R}$ parietal & L moderate & ca 290 \\
\hline N2 & $59 / \mathrm{F}$ & $\begin{array}{l}\text { Grade III } \\
\text { glioma }\end{array}$ & $\begin{array}{l}\mathrm{R} \text { thalamus with } \\
\text { temporoparietal } \\
\text { edema }\end{array}$ & L moderate & ca 510 \\
\hline N3 & $44 / F$ & Infarct & R parietal & L severe & 125 \\
\hline C1 & $60 / \mathrm{F}$ & Haemorrhage & L basal ganglia & $\mathrm{R}$ moderate & 14 \\
\hline $\mathrm{C} 2$ & 33/M & Infarct & R temporal & - & 6 \\
\hline C3 & $45 / \mathrm{M}$ & $\begin{array}{l}\text { OP grade } \\
\text { IV glioma }\end{array}$ & $\mathrm{R}$ temporal & L moderate & 54 \\
\hline $\begin{array}{l}\text { C4 } \\
\text { C5 }\end{array}$ & $\begin{array}{l}55 / M \\
68 / M\end{array}$ & $\begin{array}{l}\text { Infarct } \\
\text { Lymphoma }\end{array}$ & $\begin{array}{l}\mathrm{L} \text { frontotemporal } \\
\mathrm{R} \text { frontal }\end{array}$ & $\underline{\mathrm{R}}$ moderate & $\stackrel{9}{9}$ \\
\hline C6 & $58 / \mathrm{F}$ & Infarct & $\mathrm{L}$ frontal & - & 12 \\
\hline
\end{tabular}

$\mathrm{OP}=$ Operation . velocity of $1 \%$ s. More details were reported in a previous study that used the same apparatus. ${ }^{3}$

The objective position of the body's spatial orientation was defined by laser position $0 \% 0^{\circ}$, which was aligned with the centre of the chair and the subject's body midline in the horizontal plane and the individual eye level of the patient in the sagittal plane. The testing procedure started with the laser spot being pseudorandomly presented in one of four eccentric positions $\left(+10^{\circ} \%+10^{\circ},-10^{\circ} \%-10^{\circ},+10^{\circ} \%\right.$ $-10^{\circ},-10^{\circ} \%+10^{\circ}$ ). (Directions were defined with respect to objective body position and named in the conventional way: up and right as positive, down and left as negative.) The patient then had to direct the laser to the position which was felt to lie exactly "straight ahead" of the body's orientation. No time limit was used. No feedback was provided on accuracy.

Optokinetic stimulation was evoked by a constant linear moving pattern of randomly distributed white dots of different sizes (ca $2^{\circ}-6^{\circ}$ of visual angle). The pattern covered the whole inner surface of the cabin by using a rotating perforated sphere covering a white light situated above the patient's head.

The influence of optokinetic stimulation on the "straight ahead" judgements was investigated in the following order of test conditions: (a) No optokinetic stimulation (fixed position of the dot pattern); (b) pattern movement to the left (around the earth vertical axis); (c) pattern movement to the right. The angular velocity of the movement was $30 \%$ in both directions.

For statistical analysis a repeated analysis of variance (ANOVA) was carried out which analysed the effect of "test condition" as a within subject fixed effect. ated directly above the patient's head. The laser point was directed by the experimenter according to the instructions (up/down/ left/right) given by the patients. It moved smoothly in the indicated directions with a

\author{
Figure 1 Subjective \\ "straight ahead" \\ judgements (horizontal \\ plane) in the three test \\ conditions performed by the \\ three patients with neglect; \\ (diamonds $=N 1$; triangles \\ =N2; squares $=N 3$ ); no \\ $O K S=$ no optokinetic \\ stimulation; leftward OKS \\ $=$ leftward optckinetic \\ stimulation; rightward \\ OKS = rightward \\ optokinetic stimulation.
}

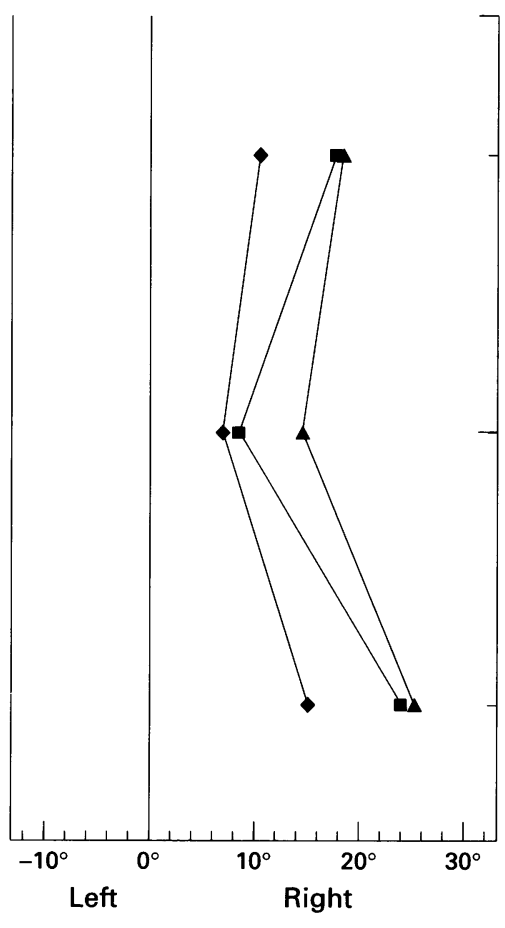

\section{No}

OKS

Leftward

OKS

Rightward OKS

\section{Results}

With the dot pattern fixed, the subjective "straight ahead" judgements of the three patients with neglect differed considerably in the horizontal plane from the objective body position and was displaced to the ipsilesional right side (fig 1). By contrast, subjective body orientation of the two control groups were closely scattered around the objective body position (fig 2).

During optokinetic stimulation all patients perceived self motion. The direction of apparent motion was to the right with leftward movement of the dot pattern and to the left with rightward movement. Figures 1 and 2 show the judgements of the subjective "straight ahead" body orientation for the three patients with neglect and the two control groups in the horizontal plane. With leftward movement of the surround the patients with neglect showed a decrease of their displaced judgement of body orientation whereas rightward movement further increased it. In the control groups, optokinetic stimulation led to a horizontal displacement of the subjective body orientation to the left with moving the dot pattern to the left and to the right with a movement to the right. 
Figure 2 Subjective "straight ahead" judgements (horizontal plane) in the three test conditions performed by the two control groups; filled circles $=$ brain damaged patients; open circles $=$ non-brain damaged patients; judgements of each patient (left) and respective mean values (right); no OKS = no optokinetic stimulation; leftward $O K S$ = leftward optokinetic stimulation; rightward $O K S=$ rightward optokinetic stimulation.
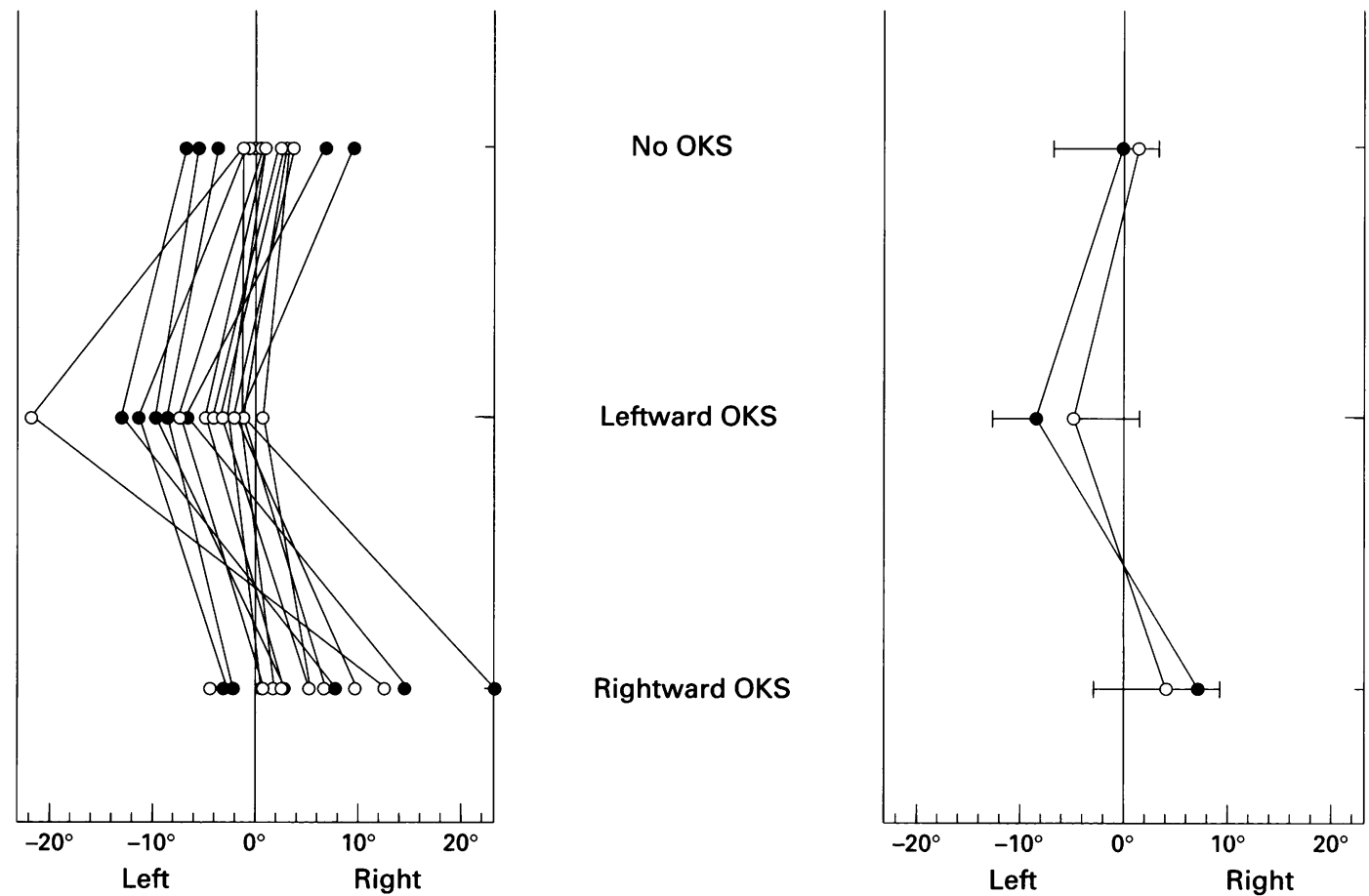

Statistical analysis of "straight ahead" judgements in the horizontal plane showed significant main effects for both factors $(F(2,16)=16 \cdot 13, \mathrm{P}<0 \cdot 000 / F(2,32)=23 \cdot 80$, $P<0.000)$. Post hoc comparison of the group factor using a corrected $\alpha$ level $^{5}$ showed a significant deviation of the horizontal "straight ahead" position to the right for the patients with neglect compared with both control groups $(t=5.82, \quad \mathrm{P}=0.017 / t=4.40, \quad \mathrm{P}=$ $0.005)$. Post hoc comparisons of factor "test condition" showed significant differences between each of the test conditions (paired samples $t$ test: $t_{1 / 2}=6.03, \quad \mathrm{P}<0.000 / t_{1 / 3}=$ $-3.50, \mathrm{P}=0.003 / t_{2 / 3}=-5.59, \mathrm{P}<0.000$ ).

In the sagittal plane, the "straight ahead" judgements of the brain damaged patients (those with and those without neglect) were comparable with those of the non-brain damaged group and closely scattered around the objective eye level in each of the three test conditions.

\section{Discussion}

Two recent studies reported an influence of optokinetic stimulation on deficient task performance in patients with neglect. Pizzamiglio et $a l^{6}$ asked non-brain damaged subjects and patients with right brain damage, with and without neglect, to bisect lines either in the presence of a stationary or a moving background. Under the moving condition a displacement of the subjective midpoint in the direction of the movement was seen in all three groups. In the group of patients with neglect, optokinetic stimulation with leftward movement of the background could reduce the displacements of bisection marks, whereas optokinetic stimulation to the right markedly increased the displacement.

Vallar $e t ~ a l^{7}$ found positive effects with optokinetic stimulation on the deficient sense of forearm position in patients with right brain damage and neglect. The forearm (not visible for the subject) was moved by the examiner to one of four different locations in the horizontal plane. The patients had to determine the selected forearm position. Compared with patients with right or left brain damage without neglect and normal controls, position judgements of the patients with neglect were more severely impaired with both the contralateral and the ipsilateral arm. Optokinetic stimulation with a leftward movement improved the deficit in forearm position sense, whereas stimulation with a rightward movement produced a worsening of the patients' performance.

The authors of both studies hypothesised that an ipsilateral displacement of the egocentric representation of the body may explain the observed effects of optokinetic stimulation. The present findings are in favour of this assumption, although further studies have to clarify possible interactive effects of different motion in patients with neglect. ${ }^{8}$ In agreement with previous findings, ${ }^{1-39}$ our patients with neglect showed a pronounced deviation of subjective body orientation toward the ipsilesional right. Optokinetic stimulation with a horizontally moving surround towards the contralesional side positively influenced their whereas a movement to the right worsened the displacement of subjective straight ahead position.

The results support the hypothesis that the mechanism leading to spatial neglect-that is, the patients' deficient exploration and response to stimuli in the contralesional part of space-is due to a disturbed transformation of input coordinates from the peripheral senprocessing mechanisms induced by visual disturbed perception of body orientation, 
sory organs into an egocentric coordinate frame of reference. ${ }^{3}$ In patients with neglect this coordinate transformation works with a systematic error and deviation of the spatial reference frame to the ipsilesional side leading to a corresponding displacement of subjective localisation of body orientation.

The present data, together with those previously reported, ${ }^{3}$ suggest that the afferent information of visual input, together with vestibular and neck proprioceptive input, is used for computing a unitary central representation of egocentric space. Interaction of the contributing input channels is used for object motion detection and dynamic spatial orientation. ${ }^{1011}$ Furthermore, the egocentric representation allows us to correctly determine our body position in space $^{12}$ and serves as a matrix for visuomotor coordination and exploration of space. ${ }^{13}$

Similar to the compensation of spatial neglect by neck muscle vibration and by caloric vestibular stimulation, the positive effect of optokinetic stimulation in patients with neglect is interpreted with a central "correction" of the neural coordinate transformation process by producing asymmetric input at the sensory organs of the contributing channels (here by applying a large field optokinetic stimulus causing visual to vestibular convergence already at the level of the brainstem). It is the asymmetric stimulation of these input channels that induces apparent self motion and the feeling of a displacement of body orientation in space.

Thus, in normal subjects the same stimulation should lead to a transient disparity of subjective and objective body orientation. In fact this was found with optokinetic, ${ }^{4}$ vestibular, ${ }^{1 /}$ and neck proprioceptive stimulation. ${ }^{12}$ Further, a displaced localisation of the body's orientation should result when the asymmetric input is induced by a disturbed primary perception of stimuli via these information channels. Indeed, this was found-for example, in patients with acute unilateral peripheral vestibular disorder, ${ }^{15}$ in patients with hemi- anopia, ${ }^{16}$ and in normal subjects after a prolonged exposure to prismatic displacement of the visual scene. ${ }^{17}$

This investigation was supported by the Deutsche Forschungsgemeinschaft and the Bundesministerium für Bildung, Wissenschaft, Forschung und Technologie. I thank Bildung, Wissenschaft, Forschung und Technologie. I thank
Professor Dichgans and the two reviewers for helpful discussion.

1 Heilman KM, Bowers D, Watson RT. Performance on hemispatial pointing task by patients with neglect syndrome. Neurology 1983;33:661-4.

2 Mark VW, Heilman KM Bodily neglect and orientational biases in unilateral neglect syndrome and normal subjects. Neurology 1990;40:640-3.

3 Karnath H-O. Subjective body orientation in neglect and the interactive contribution of neck muscle proprioception and vestibular stimulation. Brain 1994;117:1001-12.

4 Brecher GA, Brecher $\mathrm{MH}$, Kommerell G, Sauter FA, Sellerbeck J. Relation of optical and labyrinthean orientation. Opt acta 1972;19:467-71.

5 Holm S. A simple sequentially rejective multiple test procedure. Scand $\mathcal{F}$ Stat 1979;6:65-70.

6 Pizzamiglio L, Frasca R, Guariglia C, Incoccia C, Antonucci G. Effect of optokinetic stimulation in patients with visual neglect. Cortex 1990;26:535-40.

7 Vallar G, Antonucci G, Guariglia C, Pizzamiglio L. Deficits of position sense, unilateral neglect and optokinetic stimulation. Neuropsychologia 1993;31:1191-200.

8 Mattingley JB, Bradshaw JL, Bradshaw JA. Horizontal visual motion modulates focal attention in left unilateral visual motion modulates focal attention in left unilateral
spatial neglect. 7 Neurol Neurosurg Psychiatry 1994;57: $1228-35$.

9 Chokron S, Imbert M. Variations of the egocentric reference among normal subjects and a patient with unilateral neglect. Neuropsychologia 1995;33:703-11.

10 Dichgans J, Brandt T. Visual-vestibular interaction: effects on self-motion perception and postural control. In: Held R, Leibowitz HW, Teuber H-L, eds. Handbook of sensory physiology. Vol VIII. Perception. Berlin: Springer, 1978 physiology.

11 Mergner T, Rottler G, Kimmig H, Becker W. Role of vestibular and neck inputs for the perception of object motion in space. Exp Brain Res 1992;89:655-68.

12 Jeannerod $M$, Biguer $B$. The directional coding of reaching movements. A visual conception of spatial neglect. In: M Jeannerod, ed. Neuropsychological and neurophysiological aspects of spatial neglect. Amsterdam: Elsevier, aspects of spat $1987: 87-113$.

13 Karnath $\mathrm{H}-\mathrm{O}$, Fetter $\mathrm{M}$. Ocular space exploration in the dark and its relation to subjective and objective body orientation in neglect patients with parietal lesions. Neuropsychologia 1995;33:371-7.

14 Fischer MH, Kornmüller AE. Egozentrische Lokalisation 2. Mitteilung (optische Richtungslosigkeit beim vestibulären Nystagmus). I Psychol Neurol (Lpz) 1931 41:383-420

15 Hörnsten G. Constant error of visual egocentric orientation in patients with acute vestibular disorder. Brain 1979, 102:685-700.

16 Fuchs W. Untersuchung über das Sehen der Hemianopiker und Hemiamblyopiker: I. Verlagerungserscheinungen. $Z$ Psychol Physiol Sinnerorg 1920;84:67-169.

17 Held R Bossom J Neonatal deprivation and adult rearrangement: complementary techniques for analyzing plastic sensory-motor coordinations. f Comp Physiol Psychol 1961;54:33-7. 